\title{
On the Economically Optimal Exploitation of a Renewable Resource: The Case of a Convex Environment and a Convex Return Function*
}

\author{
Herbert Dawid \\ Institute of Management Science, University of Vienna, A-1210 Vienna, Austria
}

and

Michael Kopel

Department of Managerial Economics and Industrial Organization, University of Technology, A-1040 Vienna, Austria

Received November 1, 1995; revised February 6, 1997

\begin{abstract}
We investigate optimal policies for the exploitation of a renewable resource subject to a concave growth function and a convex return function. We prove an important result on the elasticity of the objective function and apply it to give a characterization of the optimal harvesting paths in this class of models. In particular, we derive conditions such that all optimal programs converge to a fixed point or to a cycle of finite period, and hence result in the conservation of the resource. We also show which assumptions guarantee that extinction of the stock is optimal. That nonconvex preferences or technologies cause problems for economic theory is well-known. As our model can formally be interpreted as an optimal growth model with nonconcave utility function, our results are a further contribution on the dynamics of optimal paths in the general class of economic models with nonconcavities. Journal of Economic Literature Classification Numbers: C61, E32, Q20. (C) 1997 Academic Press
\end{abstract}

\section{INTRODUCTION}

The problem of optimal resource exploitation has been analyzed in some detail in the literature on biological and economic systems. Clark [6] assumed a linear return (or net benefit) function and investigated the dynamics of optimal harvesting paths for a concave and an "S-shaped"

* We are grateful for helpful comments of J. Conrad, D. Easley, T. Mitra, K. Shell, and H. Wan Jr. This paper was written while M. Kopel was visiting the Department of Economics, Cornell University. Financial support of the Austrian Science Foundation under Contracts J01003-SOZ and P7783-PHY is gratefully acknowledged. 
reproduction (or growth) function; see also [23, 25]. In the first case he showed that if the marginal growth rate of the stock is always less than the reciprocal of the discount factor, any optimal path converges to zero, and hence an extinction program is optimal for every initial stock. If the marginal growth rate is larger than the reciprocal of the discount factor for some stock, there exists a (unique) steady state and it is optimal to attain it, and to remain there; hence, a sustained yield policy is optimal for every initial stock. The long term optimal behavior is independent of the initial stock. In the case of a nonconcave growth function for "sufficiently mild" and "sufficiently strong" discounting the same behavior as in the concave case is optimal. Additionally, Clark [6] and Majumdar and Mitra [23] showed that there exists an intermediate range for the discount factor (intermediate discounting), where the optimal harvesting policy depends on the initial stock. Majumdar and Mitra [24] and Dechert and Nishimura [11] showed that the results derived for a linear net benefit function and nonconcave growth function still hold for a strictly concave net benefit function. In a recent paper Olson and Roy [30] consider the case where the immediate return from harvesting depends explicitly on the stock and derive conditions for this type of model which guarantee either the optimality of a conservation or an extinction policy.

Whereas the type of nonconcavity mentioned above arises in the technology (or the biological growth curve), a second type of nonconcavity may arise in the net benefit function. In the present paper we focus on this type of nonconcavity and consider a renewable resource model in which the return function is strictly convex in the amount harvested. The growth of the resource is governed by a concave, piecewise linear, natural reproduction function, but restricted by a natural carrying capacity of the environment (for such a type of reproduction function, see e.g. [32]; Nishimura and Yano [29] derive a growth function of a similar shape in the context of a Leontief technology). The assumption of a convex return function is nonstandard in economics, but it is relevant from an empirical and theoretical point of view, e.g., in resource economics. Recently, Bjørndal et al. [5] used data from Norwegian fishery and estimated the parameters of a cost function of the Cobb-Douglas type. In all estimations they found parameter values indicating increasing returns, "which may be due to boats sharing information about locations of seals." ([5], p. 165); see also [3, 4]. Similar results were found earlier by Hannesson [15] in an empirical investigation of fisheries with data covering the years 1950-78. If we interpret the objective function as representing the utility gained from harvesting, the assumption of convexity is equivalent with the assumption of a risk taking decision maker. An argument in favor of this view is given by Conrad and Clark [9], who remark that "many commentators suggest that fishermen are risk takers." (p. 190). The empirical evidence given 
above strongly supports increasing returns in fishery economics, but there is also evidence for this assumption in other fields in economics. If one assumes linear sales revenues, the convexity of the net benefit function reduces to the concavity of the cost function. In the field of advertising, empirical evidence suggests that, e.g. due to the presence of quantity discounts on increased purchases, the marginal costs are falling (see e.g. [ 8 , 10, 34]. Furthermore, there may be fixed or set-up costs in each period which lead to a nonconcave return function.

A theoretical analysis of a model with fixed costs was carried out by Levhari et al. [22] who stress the importance of the latter for economic considerations and illustrate the relationship between nonconcavities in the population dynamics and nonconcavities in the objective function. The case of set-up costs in discrete-time models has been analyzed in [16, 31, 37]. In contrast to the case of a nonconcavity arising in the reproduction curve, where all optimal paths are monotonic, with nonconvex costs cyclical policies might be optimal (see also [14, 18, 19]). For analyses of continuous-time models with nonconcave production functions we refer the reader to $[20,35,39]$.

As the empirical and theoretical evidence given above suggest, the case of a convex return function is indeed relevant for economic considerations. Although the analysis, by relaxing the standard assumption of the concavity of the return function, is quite cumbersome from a mathematical point of view, we are able to give a complete characterization of the optimal policies for the case where the growth rate is larger than the discount rate. In particular, we prove that the optimal policy is characterized by a transient phase depending on the initial stock, and a stationary cyclic phase. Cyclical harvesting strategies of this type are often referred to as "pulsed fishing" strategy, and can be observed in real-world situations (see e.g. [7]). This shows that not only the assumptions but also the results are in line with empirical evidence. To determine the actual period of the stationary cycle, we present a simple method. In the case where the growth rate of the stock is smaller than the discount rate we are able to give a rather complete characterization except in some pathological cases. In this case extinction of the stock is indicated as being optimal, where we give criteria in order to distinguish between immediate and eventual extinction.

The paper is organized as follows. In Section 2 we present the model and give some definitions from the theory of dynamic optimization. In Section 3 we present some general results on the properties of the optimal policy which hold independent of the values of discount and growth rate. We also prove a result which holds for any function with continuous elasticity and, although of considerable interest for its own, turns out to be a crucial tool in the subsequent analysis of the model. The case where the growth rate is larger than the discount rate-the supramarginal case-is covered in 
Section 4 and in Section 5 we deal with the counterpart, the submarginal case. We end the paper with a discussion of the results in Section 6.

\section{THE MODEL}

Let $x_{t}$ denote the stock of some centrally managed renewable resource which may be harvested by a central planner at any discrete time $t=0, \ldots, \infty$. There exists a natural carrying capacity of $M>0$ which denotes the maximal stock size possible, and until the stock has reached this size the resource grows linearly with a rate of $\lambda, \lambda>0$. As no negative stocks of $x$ are admitted the harvest at time $t$ denoted by $u_{t}$ has to satisfy $0 \leqslant u_{t} \leqslant \min \left[(1+\lambda) x_{t}, M\right]$. The immediate return from a harvest $u$ is given by $R(u)$, where $R(\cdot)$ is nonnegative, strictly increasing and strictly convex which would e.g. follow from the assumption of constant prices and concave harvesting costs, where the prices are larger than marginal costs. The central planner tries to maximize the sum of discounted net benefits, where $\delta \in(0,1)$ is the discount factor. This gives the model

$$
\begin{aligned}
& \operatorname{Max} \sum_{t=0}^{\infty} \delta^{t} R\left(u_{t}\right) \\
& x_{t+1}=\min \left[M,(1+\lambda) x_{t}\right]-u_{t}, \quad x_{t}, u_{t} \geqslant 0 \quad \forall t \geqslant 0, \\
& \delta \in(0,1), \quad \lambda, M>0, \quad R \in C^{2}[0, M], \quad R(0)=0, \quad R^{\prime}(u)>0, \\
& R^{\prime \prime}(u)>0, \quad \forall u \in(0,1] .
\end{aligned}
$$

In what follows we assume without restriction of generality that $M=1$. In the rest of the paper we will characterize the dynamics of optimal paths in this model. In order to facilitate the understanding of our results we first present some basic definitions and results from the theory of dynamic optimization.

Define a set $\Omega \subset[0,1]^{2}$ as follows

$$
\Omega=\left\{\left(x, x^{\prime}\right) \in[0,1]^{2}: x^{\prime} \leqslant \min [(1+\lambda) x, 1]\right\} .
$$

We call a sequence $\left\{x_{t}\right\}_{t=0}^{\infty}$ a program from $x \in[0,1]$, if $x_{0}=x$ and $\left(x_{t}, x_{t+1}\right) \in \Omega \forall t \geqslant 0$. The corresponding sequence of controls $\left\{u_{t}\right\}_{t=0}^{\infty}$ is given by $u_{t}=\min \left[(1+\lambda) x_{t}, 1\right]-x_{t+1}$. We say a sequence of controls is admissible from $x$ iff it corresponds to some program from $x$. A sequence $\left\{\hat{u}_{t}\right\}_{t=0}^{\infty}$ is called optimal if

$$
\sum_{t=0}^{\infty} \delta^{t} R\left(\hat{u}_{t}\right) \geqslant \sum_{t=0}^{\infty} \delta^{t} R\left(u_{t}\right)
$$


holds for every sequence of controls $\left\{u_{t}\right\}_{t=0}^{\infty}$ admissible from $x$. The corresponding program $\left\{\hat{x}_{t}\right\}_{t=0}^{\infty}$ is called an optimal program from $x$.

The function $W:[0,1] \mapsto \mathbb{R}_{+}$defined by

$$
W(x)=\sum_{t=0}^{\infty} \delta^{t} R\left(\hat{u}_{t}\right)
$$

for some optimal sequence of controls $\left\{\hat{u}_{t}\right\}_{t=0}^{\infty}$ from $x$ is called the value function. Under our assumptions the value function is unique and the unique bounded solution of the Bellman equation

$$
W(x)=\max _{x^{\prime}:\left(x, x^{\prime}\right) \in \Omega}\left[R\left(\min [(1+\lambda) x, 1]-x^{\prime}\right)+\delta W\left(x^{\prime}\right)\right] .
$$

The map $\theta:[0,1] \mapsto \mathscr{P}([0,1])$ given by

$$
\theta(x)=\left\{x^{\prime} \in[0,1]: x^{\prime}=\hat{x}_{1} \text { for some optimal program }\left\{\hat{x}_{t}\right\}_{t=0}^{\infty} \text { from } x\right\}
$$

is called the optimal policy. In general, $\theta(x)$ will be set-valued and upper semi continuous. Note, however, that if the optimal policy is single-valued this implies the uniqueness of all optimal programs. Obviously $y \in \theta(x)$ implies

$$
y \in \underset{x^{\prime}:\left(x, x^{\prime}\right) \in \Omega}{\arg \max }\left[R\left(\min [(1+\lambda) x, 1]-x^{\prime}\right)+\delta W\left(x^{\prime}\right)\right] .
$$

We refer to Stokey and Lucas [38] for more details on dynamic optimization problems.

\section{GENERAL RESULTS}

In this section we derive some results which hold for all feasible values of $\delta$ and $\lambda$. They will prove to be useful for the further analysis in Sections 4 and 5. The first lemma shows that both the value function and the optimal policy are constant on the interval $[1 /(1+\lambda), 1]$.

Lemma 1. Let $W(x)$ denote the value function and $\theta(x)$ denote the optimal policy, then

$$
W(x)=W\left(\frac{1}{(1+\lambda)}\right)
$$


and

$$
\theta(x)=\theta\left(\frac{1}{(1+\lambda)}\right)
$$

holds for all $x \geqslant 1 /(1+\lambda)$.

Proof. Let $x \geqslant 1 /(1+\lambda)$. The value function has to satisfy the Bellman equation

$$
W(x)=\max _{y \in[0,1]}[R(1-y)+\delta W(y)]
$$

The value $z \in[0,1]$ is an element of the optimal policy $\theta(x)$ if and only if it satisfies

$$
z \in \underset{y \in[0,1]}{\operatorname{argmax}}[R(1-y)+\delta W(y)] .
$$

As $x$ does not appear in the right hand side of either of the equations the lemma follows immediately.

As a corollary of Lemma 1 it follows that any element of the optimal policy is less or equal $1 /(1+\lambda)$. This seems reasonable as the building up of the resource stock larger than $1 /(1+\lambda)$ implies that not the full potential of growth of the resource can be used in the next period.

Corollary 1. For any $x \in[0,1]$,

$$
z \in \theta(x) \Rightarrow z \leqslant \frac{1}{(1+\lambda)} .
$$

The next Lemma is of crucial importance for our further results.

Lemma 2. Each selection of the optimal policy is non-increasing on the interval $\left[1 /(1+\lambda)^{2}, 1\right]$.

Proof. We know from Lemma 1 that $\theta(x)$ is constant on $[1 /(1+\lambda), 1]$. Hence it suffices to show this property of $\theta(x)$ for the interval $\left[1 /(1+\lambda)^{2}\right.$, $1 /(1+\lambda))$. Let us assume there exist two values $x<y$ in $\left[1 /(1+\lambda)^{2}\right.$, $1 /(1+\lambda)]$ and two values $\tilde{x}, \tilde{y}$ with $\tilde{x} \in \theta(x), \tilde{y} \in \theta(y)$ and $\tilde{x}<\tilde{y}$. According to Corollary 1 we have $\tilde{x}, \tilde{y} \leqslant 1 /(1+\lambda)$. Define $g(z)$ as

$$
g(z)=R((1+\lambda) z-\tilde{x})-R((1+\lambda) z-\tilde{y})+\delta(W(\tilde{x})-W(\tilde{y}))
$$


From $\tilde{x} \in \theta(x)$ we conclude that $g(x) \geqslant 0$ must hold. Differentiation yields

$$
\begin{gathered}
g^{\prime}(z)=(1+\lambda)\left(R^{\prime}((1+\lambda) z-\tilde{x})-R^{\prime}((1+\lambda) z-\tilde{y})\right)>0, \\
\forall z \in\left[\frac{1}{(1+\lambda)^{2}}, \frac{1}{1+\lambda}\right]
\end{gathered}
$$

as $R(x)$ is strictly convex. This implies

$$
R((1+\lambda) y-\tilde{x})+\delta W(\tilde{x})>R((1+\lambda) y-\tilde{y})+\delta W(\tilde{y}) .
$$

Together with $\tilde{y} \in \theta(y)$ we get a contradiction.

We would like to point out the connection between this result and the well-known result of Benhabib and Nishimura [2] on the dynamics of optimal paths in the interior of the feasible set. There are, however, two differences: first, we deal with a convex objective function and second, we do not restrict ourselves to interior paths (see also [28]).

The next lemma shows that due to the convexity of $R(u)$ it is optimal to exploit the resource only once between those periods where the stock of resource lies on the boundary of the admissible interval $[0,1]$.

Lemma 3. Let $\left\{\hat{x}_{t}\right\}_{t=0}^{\infty}$ be an optimal program from $x \in[0,1]$ and let $\left\{\hat{u}_{t}\right\}_{t=0}^{\infty}$ be the corresponding sequence of optimal controls. Then there is an index $t$ such that $\hat{x}_{t}=1 /(1+\lambda)$ or $\hat{x}_{t}=0$. Let $\hat{n}$ be the smallest of these indices, then there is at most one $\hat{u}_{t}$ with $t<\hat{n}$ and $\hat{u}_{t} \neq 0$.

Proof. Due to Corollary $1,0 \leqslant \hat{x}_{t} \leqslant 1 /(1+\lambda) \forall t$. Assume that there is no index $t$ with $\hat{x}_{t}=1 /(1+\lambda)$. This implies that the dynamics of the state variable is given by

$$
\hat{x}_{t+1}=(1+\lambda) \hat{x}_{t}-\hat{u}_{t}
$$

Assume further that there are two indices $0 \leqslant n_{1}<n_{2}$ with $\hat{u}_{n_{1}}>0, \hat{u}_{n_{2}}>0$, $\hat{u}_{t}=0 \forall t<n_{2}, t \neq n_{1}$ (note that this implies $\hat{u}_{n_{1}}<(1+\lambda) \hat{x}_{n_{1}}$ ). Obviously the value function of $x$ is in this case given by

$$
W(x)=\delta^{n_{1}} R\left(\hat{u}_{n_{1}}\right)+\delta^{n_{2}} R\left(\hat{u}_{n_{2}}\right)+\delta^{\left(n_{2}+1\right)} W\left(\hat{x}_{n_{2}+1}\right) .
$$

Now consider the following sequence of controls: $\tilde{u}_{t}=\hat{u}_{t} \forall t \neq n_{1}, n_{2}$ and $\tilde{u}_{n_{1}}=\hat{u}_{n_{1}}+y, \tilde{u}_{n_{2}}=\hat{u}_{n_{2}}-(1+\lambda)^{\left(n_{2}-n_{1}\right)} y$. It is easy to check that the corresponding program $\left\{\tilde{x}_{t}\right\}_{t=0}^{\infty}$ is admissible if $y$ is positive and sufficiently small and that 


$$
\begin{aligned}
\tilde{x}_{n_{2}+1} & =(1+\lambda)^{n_{2}-n_{1}} \tilde{x}_{n_{1}+1}-\tilde{u}_{n_{2}} \\
& =(1+\lambda)^{n_{2}-n_{1}}\left((1+\lambda) \hat{x}_{n_{1}}-\left(\hat{u}_{n_{1}}+y\right)\right)-\left(\hat{u}_{n_{2}}-(1+\lambda)^{n_{2}-n_{1}} y\right) \\
& =(1+\lambda)^{n_{2}-n_{1}} \hat{x}_{n_{1}+1}-(1+\lambda)^{n_{2}-n_{1}} y-\hat{u}_{n_{2}}+(1+\lambda)^{n_{2}-n_{1}} y \\
& =(1+\lambda) \hat{x}_{n_{2}}-\hat{u}_{n_{2}}=\hat{x}_{n_{2}+1} .
\end{aligned}
$$

Therefore the discounted payoff of such a program is given by

$$
g(x, y)=\delta^{n_{1}} R\left(\hat{u}_{n_{1}}+y\right)+\delta^{n_{2}} R\left(\hat{u}_{n_{2}}-(1+\lambda)^{\left(n_{2}-n_{1}\right)} y\right)+\delta^{\left(n_{2}+1\right)} W\left(\hat{x}_{n_{2}+1}\right) .
$$

We have $W(x)=g(x, 0)$ and as $W(x)$ is the value function, the conditions $\partial / \partial y g(x, 0)=0, \partial^{2} / \partial y^{2} g(x, 0)<0$ must hold. Differentiation yields however

$$
\frac{\partial^{2}}{\partial y^{2}} g(x, 0)=\delta^{n_{1}} R^{\prime \prime}\left(\hat{u}_{n_{1}}\right)+\delta^{n_{2}}(1+\lambda)^{2\left(n_{2}-n_{1}\right)} R^{\prime \prime}\left(\hat{u}_{n_{2}}\right)>0 .
$$

We get a contradiction, which implies that our assumption must have been wrong. Thus only one positive control may appear if $\hat{x}_{t}<1 /(1+\lambda) \forall t$ which means that there has to exist an index $\hat{n} \geqslant 0$ with $\hat{u}_{t}=0 \forall t \neq \hat{n}-1$, $\hat{u}_{\hat{n}-1}=(1+\lambda) \hat{x}_{\hat{n}-1}$ which corresponds to an optimal program of the form $\hat{x}_{t+1}=(1+\lambda) \hat{x}_{t} \forall t<\hat{n}-1, \hat{x}_{t}=0 \forall t \geqslant \hat{n}$. On the other hand if there exists some $\hat{n}$ with $\hat{x}_{\hat{n}}=1 /(1+\lambda), \hat{x}_{t} \neq 1 /(1+\lambda) \forall t<\hat{n}$ the same argument as above shows that there is at most one positive control $\hat{u}_{t}>0$ with $t<\hat{n}$.

Lemma 4 gives a general result which holds for any function $R$ with continuous elasticity. It says that if we change the argument of the function by a factor $c>1$, the value of the function changes by a factor of $c$ to the power of the average elasticity of $R(u)$ in the interval $[u, c u]$. Although the derived formula is very useful, we could not find it in the economics literature. Whereas this result is of interest in its own, it will prove to be a crucial tool in our analysis.

Lemma 4. Denote by

$$
\varepsilon(u)=\frac{R^{\prime}(u) u}{R(u)}
$$

the elasticity of $R(u)$. Then for any $c>1$

$$
R(c u)=c^{(1 /(c-1) u) \int_{u}^{c u} \varepsilon(z) d z} R(u)
$$

holds for all $u \in[0,1 / c]$. 
Proof. Define a sequence $\left.\{\mu(n)\}_{n=1}^{\infty}\right\}$ as $\mu(n)=\sqrt[n]{c}$ and for each $n$ define $u_{i}(n)=u \mu(n)^{i}, i=0, \ldots, n$. First we show that $\lim _{n \rightarrow \infty} \prod_{i=0}^{n-1} \mu(n)^{\varepsilon\left(u_{i}(n)\right)}=$ $R(c u) / R(u)$. We have

$$
\begin{aligned}
& \frac{1}{\lim _{n \rightarrow \infty} \prod_{i=0}^{n-1} \mu(n)^{\varepsilon\left(u_{i}(n)\right)}} \frac{R(c u)}{R(u)} \\
& =\lim _{n \rightarrow \infty}\left[\left(\prod_{i=0}^{n-1} \frac{1}{\mu(n)^{\varepsilon\left(u_{i}(n)\right)}}\right)\left(\prod_{i=0}^{n-1} \frac{R\left(\mu(n) u_{i}(n)\right)}{R\left(u_{i}(n)\right)}\right)\right] \\
& =\lim _{n \rightarrow \infty} \prod_{i=0}^{n-1} \phi\left(u_{i}(n), n\right),
\end{aligned}
$$

where $\phi(z, n)=R(\mu(n) z) / R(z) \mu(n)^{\varepsilon(z)}$. Next we show that $\lim _{n \rightarrow \infty} \phi(z, n)^{n}$ $=1 \forall z \in[u, c u]$. This can be seen by calculating

$$
\begin{aligned}
\lim _{n \rightarrow \infty} \ln \left(\phi(z, n)^{n}\right) & =\lim _{n \rightarrow \infty}\left(n \ln \left(\frac{R(\mu(n) z)}{R(z)}\right)-\varepsilon(z) \ln \left(\mu(n)^{n}\right)\right) \\
& =\frac{R^{\prime}(z) z}{R(z)} \ln (c)-\varepsilon(z) \ln (c)=0,
\end{aligned}
$$

where the expression in the third line is obtained by using the rule of de l'Hospital. As the interval $[u, c u]$ is compact, the convergence is uniform and we have $\lim _{n \rightarrow \infty} \phi\left(z_{n}, n\right)^{n}=1$ for every sequence $\left\{z_{n}\right\}_{n=0}^{\infty}$ with $z_{n} \in[u, c u] \forall n \geqslant 0$. Define the sequences $\left\{\bar{z}_{n}\right\}_{n=0}^{\infty}$ and $\left\{\underline{z}_{n}\right\}_{n=0}^{\infty}$ by

$$
\begin{aligned}
& \bar{z}_{n}=\operatorname{argmax}_{z \in[u, c u]} \phi(z, n) \\
& \underline{z}=\underset{z \in[u, c u]}{\operatorname{argmin}} \phi(z, n) .
\end{aligned}
$$

We have

$$
\phi\left(\underline{z}_{n}, n\right)^{n} \leqslant \prod_{i=0}^{n-1} \phi\left(u_{i}(n), n\right) \leqslant \phi\left(\bar{z}_{n}, n\right)^{n} \quad \forall n \geqslant 0
$$

This implies $\lim _{n \rightarrow \infty} \prod_{i=0}^{n-1} \phi\left(u_{i}(n), n\right)=1$ and thus $\lim _{n \rightarrow \infty} \prod_{i=0}^{n-1} \mu(n)^{\varepsilon\left(u_{i}(n)\right)}$ $=R(c u) / R(u)$. Therefore

$$
\begin{aligned}
\frac{R(c u)}{R(u)} & =\lim _{n \rightarrow \infty} \prod_{i=0}^{n-1} \mu(n)^{\varepsilon\left(u_{i}(n)\right)} \\
& =c^{\lim _{n \rightarrow \infty}(1 / n) \sum_{i=0}^{n-1} \varepsilon\left(u_{i}(n)\right)} \\
& =c^{1 /((c-1) u) \int_{u}^{c u} \varepsilon(z) d z}
\end{aligned}
$$


Note that replacing the limit of the sum by an integral is admissible as our assumptions guarantee that $\varepsilon(z)$ is continuous in $z$.

With the use of this formula we get

LEMMA 5. Let

$$
\frac{1}{\lambda u} \int_{u}^{(1+\lambda) u} \varepsilon(z) d z>a
$$

hold for all $u \in[0,1 /(1+\lambda)]$, where

$$
a=-\frac{\ln (\delta)}{\ln (1+\lambda)},
$$

then $\theta(x)=(1+\lambda) x \forall x \in\left[0,1 /(1+\lambda)^{2}\right]$.

Proof. Let $\left\{\hat{x}_{t}\right\}_{t=0}^{\infty}$ be an optimal program from $x \in\left[0,1 /(1+\lambda)^{2}\right]$ and $\left\{\hat{u}_{t}\right\}_{t=0}^{\infty}$ the corresponding series of controls. Assume that $\hat{u}_{0}>0$. This implies by Lemma 3 that $\hat{u}_{1}=0$. Consider another program $\left\{\tilde{x}_{t}\right\}_{t=0}^{\infty}$ defined by $\tilde{u}_{0}=0, \tilde{u}_{1}=(1+\lambda) \hat{u}_{0}, \tilde{u}_{t}=\hat{u}_{t} \forall t \geqslant 2$. We get

$$
\tilde{x}_{2}=(1+\lambda)^{2} \hat{x}_{0}-(1+\lambda) \hat{u}_{0}=(1+\lambda)\left((1+\lambda) \hat{x}_{0}-\hat{u}_{0}\right)=(1+\lambda) \hat{x}_{1}=\hat{x}_{2}
$$

and thus $\tilde{x}_{t}=\hat{x}_{t} \forall t \geqslant 2$. The difference of the discounted payoffs of the two programs is therefore given by $\delta R\left((1+\lambda) \hat{u}_{0}\right)-R\left(\hat{u}_{0}\right)$. Using Lemma 4 and (3) we get by using that $\hat{u}_{0} \leqslant(1+\lambda) x \leqslant 1 /(1+\lambda)$

$$
\begin{aligned}
\delta R\left((1+\lambda) \hat{u}_{0}\right) & =\delta(1+\lambda)^{\left(1 / \lambda \hat{u}_{0}\right.}{\hat{\hat{u}_{0}}}^{\left.(1+\lambda) \hat{u}_{0} \varepsilon(z) d z\right)} R\left(\hat{u}_{0}\right) \\
& >\delta(1+\lambda)^{a} R\left(\hat{u}_{0}\right) \\
& =R\left(\hat{u}_{0}\right) .
\end{aligned}
$$

This contradicts our assumption that $\left\{\hat{x}_{t}\right\}$ is an optimal program and we thus get that $\hat{u}_{0}=0$ for every optimal program from $x$. This yields $\theta(x)=(1+\lambda) x \forall x \in\left[0,1 /(1+\lambda)^{2}\right]$.

Lemma 5 has the following economic interpretation. The parameter $a$ denotes the value of the elasticity which leaves the decision maker indifferent between harvesting an amount $u$ now and investing the revenue, or harvesting the amount of $(1+\lambda) u$ in the next period. Lemma 5 says that whenever the average elasticity in any interval $[u,(1+\lambda) u]$ is larger than this value $a$ it is optimal for the central planner to wait with exploitation of the resource as long as he can use the full potential of growth of the resource. 


\section{CONSERVATION OF RESOURCES: THE SUPRAMARGINAL CASE}

In this section we deal with the case where $\delta(1+\lambda)>1$, subsequently denoted as the supramarginal case. In the first lemma of this section we show that immediate extinction of the resource is never optimal in this setup.

Lemma 6. Assume that $\delta(1+\lambda)>1$, then $\theta(x)=(1+\lambda) x$ for all $x \in\left[0,1 /(1+\lambda)^{2}\right]$. Furthermore, $\theta(x) \neq 0$ holds for all $x \in(0,1]$.

Proof. The first part of the lemma is an immediate consequence of Lemma 5 as $\delta(1+\lambda)>1$ is equivalent with $a<1$ and the convexity of $R(u)$ implies $\varepsilon(u) \geqslant 1 \forall u \in[0,1]$. To establish the second claim of the lemma we show that $\theta(1 /(1+\lambda)) \neq 0$. Due to Lemma 3 we know that either $\theta(1 /(1+\lambda))=0$, or $\theta(1 /(1+\lambda))=1 /(1+\lambda)^{m}$ for some integer $m \geqslant 1$. This follows from the fact that no second positive control can be applied before one of the states $x=1 /(1+\lambda)$ or $x=0$ is reached. Hence, if $\theta(1 /(1+\lambda)) \neq 0$ the state $x=1 /(1+\lambda)$ has to be reached without use of any further positive control. Thus if $\theta(1 /(1+\lambda)) \neq 0$ the optimal program from $1 /(1+\lambda)$ describes a cycle with period $m$. The discounted payoff of such a cyclical program is given by $1 /\left(1-\delta^{m}\right) R\left(1-1 /(1+\lambda)^{m}\right)$. Thus we have to show that

$$
\frac{1}{1-\delta^{n}} R\left(1-\frac{1}{(1+\lambda)^{n}}\right)>R(1)
$$

holds for some $n \geqslant 1$. Defining $z=1-1 /(1+\lambda)^{n}$ the left hand side of expression (5) transforms to

$$
g(z)=\frac{1}{1-(1-z)^{a}} R(z)
$$

with $a$ given by (4) and $z \in[\lambda /(1+\lambda), 1]$. Noticing that $g(1)=R(1)$ and that for any $\eta>0$ some point $1-1 /(1+\lambda)^{n}$ lies in $(1-\eta, 1)$ we conclude that showing $g^{\prime}(z)<0$ on some interval $(1-\eta, 1)$ implies (5). Differentiating $g(z)$ we get

$$
g^{\prime}(z)=-\frac{a(1-z)^{a-1}}{\left(1-(1-z)^{a}\right)^{2}} R(z)+\frac{1}{1-(1-z)^{a}} R^{\prime}(z) .
$$

Thus $g^{\prime}(z) \rightarrow-\infty$ for $z \rightarrow 1$, if, as in our case, $a<1$ holds. Therefore, $\theta(1 /(1+\lambda)) \neq 0$ holds, which implies by Lemma 1 and Lemma 2 that $\theta(x) \neq 0$ on $\left[1 /(1+\lambda)^{2}, 1\right]$. 
Given all the results derived so far we are now able to give a general characterization of the optimal programs in the supramarginal case. This is done in our first proposition.

Proposition 1. Let $\delta(1+\lambda)>1$ and $x$ be given in $\left(1 /(1+\lambda)^{k+1}\right.$, $\left.1 /(1+\lambda)^{k}\right]$. If $k \geqslant 1$, an optimal program from $x$ has the form

$$
\begin{gathered}
\left\{x,(1+\lambda) x, \ldots,(1+\lambda)^{k-1} x, \frac{1}{(1+\lambda)^{l}}, \frac{1}{(1+\lambda)^{l-1}}, \ldots, \frac{1}{1+\lambda},\right. \\
\left.\frac{1}{(1+\lambda)^{m}}, \frac{1}{(1+\lambda)^{m-1}}, \ldots, \frac{1}{1+\lambda}, \frac{1}{(1+\lambda)^{m}}, \frac{1}{(1+\lambda)^{m-1}}, \ldots\right\} .
\end{gathered}
$$

If $k=0$ an optimal program is given by

$$
\left\{x, \frac{1}{(1+\lambda)^{m}}, \frac{1}{(1+\lambda)^{m-1}}, \ldots, \frac{1}{\lambda+1}, \frac{1}{(1+\lambda)^{m}}, \frac{1}{(1+\lambda)^{m-1}}, \ldots\right\},
$$

where $l(x)$ and $m$ are finite integers given by

$$
\begin{gathered}
m \in \underset{n \in \mathbb{N}}{\operatorname{argmax}}\left[\frac{1}{1-\delta^{n}} R\left(1-\frac{1}{(1+\lambda)^{n}}\right)\right] \\
l(x) \in \underset{n \in \mathbb{N}}{\operatorname{argmax}}\left[R\left((1+\lambda)^{k} x-\frac{1}{(1+\lambda)^{n}}\right)\right. \\
\left.+\frac{\delta^{n}}{1-\delta^{m}} R\left(1-\frac{1}{(1+\lambda)^{m}}\right)\right],
\end{gathered}
$$

and only $l(x)$ depends on the state $x$ with $l(x) \leqslant m \forall x \in(0,1 /(1+\lambda)]$.

Proof. Consider first the case $k \geqslant 1$. According to Lemma 6 we have $\hat{x}_{t}=(1+\lambda)^{t} x \quad \forall t \leqslant k-1$ and thus $\hat{x}_{k-1}=(1+\lambda)^{k-1} \quad x \in\left(1 /(1+\lambda)^{2}\right.$, $1 /(1+\lambda)]$. This implies by Corollary 1 that $\hat{u}_{k-1}$ has to be positive and by Lemmata 3 and 6 that $1 /(1+\lambda)$ has to be reached from $\hat{x}_{k}$ without the occurrence of any other positive control. Thus we get $\hat{x}_{k}=1 /(1+\lambda)^{l}$, $\hat{x}_{k+t}=1 /(1+\lambda)^{l-t}, \forall t=1, \ldots, l-1$ for some integer $l \geqslant 1$. With exactly the same arguments we conclude that $\hat{x}_{k+l}=1 /(1+\lambda)^{m}$ for some integer $m \geqslant 1$ and $\hat{x}_{k+l+t}=1 /(1+\lambda)^{m-t} \forall t=1, \ldots, m-1$. Thus any optimal program from $x$ ends up cycling and it is easy to see that the discounted payoff of these cycles is maximized if the cycle length $m$ is chosen according to (7). Thus the discounted payoff of the optimal program from $\hat{x}_{k-1}$ is given by 
the expression in the brackets on the right hand side of (8) and this implies that $l(x)$ must be given by (8) if the program is optimal. As $\hat{x}_{k-1} \leqslant 1 /(1+\lambda)$, Lemma 2 implies directly $l(x) \leqslant m$. If $k=0$ we apply Lemma 1 to conclude that $\hat{x}_{1}=\theta(1 /(1+\lambda))=1 /(1+\lambda)^{m}$.

Proposition 1 shows that in the supramarginal case every optimal program is eventually cyclic and the length of the cycle is determined by (7) independent of the initial stock. Thus each optimal program has a transient phase depending on the initial stock and a stationary cyclic phase. If we look at the corresponding optimal control sequence we realize that only one harvest takes place in the transient phase and that afterwards the same amount is harvested every $m$ th period. In the light of these findings it is of high interest to determine the actual period of the stationary cycle. In the rest of this section we give a rather simple method to achieve this. According to Proposition 1 the period of the cycle is given by (7). Using the same transformation as in the proof of Lemma 6 , namely defining $z_{n}=1-1 /(1+\lambda)^{n}$ and the function $g$ as in (6) we have to find a value $m$ such that $m=\operatorname{argmax}_{n \geqslant 1} g\left(z_{n}\right) .{ }^{1}$ Note that $z_{n}$ gives the harvest that takes place in a cycle with period $n$. The value $z_{1}=\lambda /(1+\lambda)$ corresponds to a fixed point in the program and the limit case $n=\infty$ stands for the complete extinction of the stock. Differentiating $g(z)$ we get

$$
\begin{aligned}
g^{\prime}(z) & =-\frac{a(1-z)^{a-1}}{\left(1-(1-z)^{a}\right)^{2}} R(z)+\frac{1}{1-(1-z)^{a}} R^{\prime}(z) \\
& =\frac{R(z)}{z\left(1-(1-z)^{a}\right)}\left[-\frac{a z}{\left(1-(1-z)^{a}\right)(1-z)^{1-a}}+\varepsilon(z)\right] .
\end{aligned}
$$

As the expression outside the brackets is always positive the sign of the first derivative is determined by the sign of the expression in the brackets. Thus we define

$$
\Psi_{a}(z)=\frac{a z}{\left(1-(1-z)^{a}\right)(1-z)^{1-a}} \quad \forall z \in[0,1]
$$

where the parameter $a$ is again given by (4). For all values of $z$ where $\Psi_{a}(z)<\varepsilon(z)$ the function $g(z)$ is increasing, whenever $\Psi_{a}(z)>\varepsilon(z)$ it is decreasing. Concerning the shape of $\Psi_{a}(z)$ we get the following Lemma.

Lemma 7. Let $\Psi_{a}(z)$ be defined by (9). Then $\Psi_{a}(0)=1$, and for $a<1$ the function $\Psi_{a}(z)$ is increasing on $[0,1)$ with $\lim _{z \rightarrow 1} \Psi_{a}(z)=\infty$. Furthermore, for $a>1 \Psi_{a}(z)$ is decreasing with $\Psi_{a}(1)=0$, and $\Psi_{a}(z) \equiv 1$ for $a=1$.

${ }^{1}$ We ignore here the (highly) improbable case that $\operatorname{argmax}_{n \in \mathbb{N}} g\left(z_{n}\right)$ is not unique. 
Proof. The results concerning values of the function $\Psi_{a}(z)$ at $z=0$ and $z=1$ and also the result for $a=1$ are obvious. To show the monotonicity results we differentiate $\Psi_{a}(z)$ which gives

$$
\Psi_{a}^{\prime}(z)=\frac{a}{\left(1-(1-z)^{a}\right)^{2}(1-z)^{2(1-a)}}\left((1-z)^{1-a}+\frac{(1-a) z}{(1-z)^{a}}-1\right) .
$$

Obviously the sign of $\Psi_{a}^{\prime}(z)$ is determined by the sign of the expression $h_{a}(z)=(1-z)^{1-a}+\left((1-a) z /(1-z)^{a}\right)-1$. We have $h_{a}(0)=0$. Differentiating $h_{a}$ gives

$$
h_{a}^{\prime}(z)=(1-a) \frac{a z}{(1-z)^{1+a}} .
$$

Thus $h_{a}$ is increasing and positive for $a<1$ and decreasing and negative for $a>1$.

Note that $a<(>) 1 \Leftrightarrow \delta(1+\lambda)>(<) 1$. Hence in the supramarginal case $a<1$. This implies that either $\varepsilon(z) \leqslant \Psi_{a}(z)$ holds on the entire interval $[\lambda /(1+\lambda), 1)$ or there exists at least one intersection point of $\Psi_{a}(z)$ and $\varepsilon(z)$. In the first case $g(z)$ is nonincreasing on $[\lambda /(1+\lambda), 1]$. We deal with this case later on. Now for the sake of simplicity, assume that there exists exactly one such intersection point $z^{*} \in[\lambda /(1+\lambda), 1)$, where $\varepsilon\left(z^{*}\right)=\Psi_{a}\left(z^{*}\right)$. As we have $\varepsilon(1)<\lim _{z \rightarrow 1} \Psi_{a}(1)$, the maximum of $g(z)$ in $[\lambda /(1+\lambda), 1)$ is attained at $z^{*}$ and for $z^{*} \in\left[1-\left(1 /(1+\lambda)^{n}\right), 1-\left(1 /(1+\lambda)^{n+1}\right)\right]$ the period $m$ of the stationary cycle is either $m=n$ or $m=n+1$. Therefore, the period of the long term cycle may be determined by calculating the intersection point $z^{*}$ between $\varepsilon(z)$ and $\Psi_{a}(z)$ and comparing the values $g\left(z_{n}\right)$ and $g\left(z_{n+1}\right)$, where $z_{n}<z^{*} \leqslant z_{n+1}$. The extension of this technique to cases where the two functions intersect more than once in $[\lambda /(1+\lambda), 1)$ is straightforward. We summarize these arguments in the next Proposition.

Proposition 2. Let $\delta(1+\lambda)>1$ and assume that $\varepsilon(z)$ and $\Psi_{a}(z)$ intersect at a unique point $z^{*} \in[\lambda /(1+\lambda), 1)$. Then the period $m$ of the stationary cycle of the optimal program is either given by $n$ or $n+1$, where $1-\left(1 /(1+\lambda)^{n}\right)<z^{*} \leqslant 1-\left(1 /(1+\lambda)^{n+1}\right)$.

Proof. The Proposition follows directly from the arguments given above.

In particular, the analysis above enables us to give a simple condition under which any optimal program converges to the fixed point $x=1 /(1+\lambda)$. 
COROllary 2. Let $\delta(1+\lambda)>1$ and additionally

$$
\varepsilon(z)<\Psi_{a}(z) \quad \forall z \in\left(\frac{\lambda}{1+\lambda}, 1\right)
$$

where the function $\Psi_{a}(z)$ is given by (9). Then the optimal policy is singlevalued, continuous and given by

$$
\theta(x)= \begin{cases}(1+\lambda) x & 0 \leqslant x \leqslant \frac{1}{(1+\lambda)^{2}} \\ \frac{1}{1+\lambda} & \frac{1}{(1+\lambda)^{2}}<x \leqslant 1\end{cases}
$$

The value function is given by

$$
W(x)=\left\{\begin{array}{c}
x=0 \\
\delta^{k-1} R\left((1+\lambda)^{k} x-\frac{1}{1+\lambda}\right)+\frac{\delta^{k}}{1-\delta} R\left(\frac{\lambda}{1+\lambda}\right) \\
\frac{1}{(1+\lambda)^{k+1}} \leqslant x<\frac{1}{(1+\lambda)^{k}}, \quad k \geqslant 1 \\
\frac{1}{1-\delta} R\left(\frac{\lambda}{1+\lambda}\right) \quad \frac{1}{1+\lambda} \leqslant x \leqslant 1
\end{array}\right.
$$

The integers $l(x)$ and $m$ defined by (8) and (7) are thus given by $l(x)=m=1$ $\forall x \in[0,1]$ and every optimal program converges to $x=1 /(1+\lambda)$.

Proof. We know from Lemma 6 that $\theta(x)=(1+\lambda) x$ holds for all $x \in\left[0,1 /(1+\lambda)^{2}\right]$. Taking into account Lemmata 1 and 2 we conclude that the proposition is shown if we establish $\theta(1 /(1+\lambda))=1 /(1+\lambda)$. As $\varepsilon(z)<\Psi_{a}(z) \forall z \in(\lambda /(1+\lambda), 1)$, the function $g(z)$ is nonincreasing in $[\lambda /(1+\lambda), 1)$ and attains its maximum at $z=\lambda /(1+\lambda)$. This implies $m=1$, or equivalently $\theta(1 /(1+\lambda))=1 /(1+\lambda)$. The value function in (11) can be obtained by direct calculation using the optimal policy function $\theta(x)$.

To conclude the discussion of the supramarginal case we present an example in order to illustrate the technique of finding the period $m$ of the stationary cycle. 
EXAMPLE 1. Let the immediate benefit function be given as the difference between sales revenue and concave harvesting costs

$$
R(u)=p u-q u(2-u) .
$$

The parameter $p$ denotes the market price of one unit of the resource. If $p \geqslant 2 q$ all assumptions made in (1) are fulfilled. Assume further that the parameters are given by

$$
\delta=0.9, \quad \lambda=0.2, \quad q=1 .
$$

Thus we have $a=0.577$. We will show that under these assumptions different values of $p$ imply different periods of the optimal stationary cycle. First we assume $p=10$. As demonstrated in Figure 1 the intersection point of $\Psi_{a}(z)$ and $\varepsilon(z)$ is smaller than $\lambda /(1+\lambda)$. Thus according to Corollary 2 we get a continuous optimal policy as depicted in Figure 2 and every optimal program converges to the fixed point $x=1 /(1+\lambda)=0.833$.

As a second case we deal with $p=3$. In Figure 3 we show again the two functions $\Psi_{a}(z)$ and $\varepsilon(z)$. The unique intersection point is given by $z^{*}=0.763 \in\left(1-\left(1 / 1.2^{7}\right), 1-\left(1 / 1.2^{8}\right)\right]$. Thus the period of optimal cycle is either given by 7 or 8 . Direct calculations show that it is in fact given by $m=8$. Figure 4 exhibits the optimal policy in this case. Generally speaking we realize that the period of the optimal long term cycle decreases with increasing market prices of the resource.

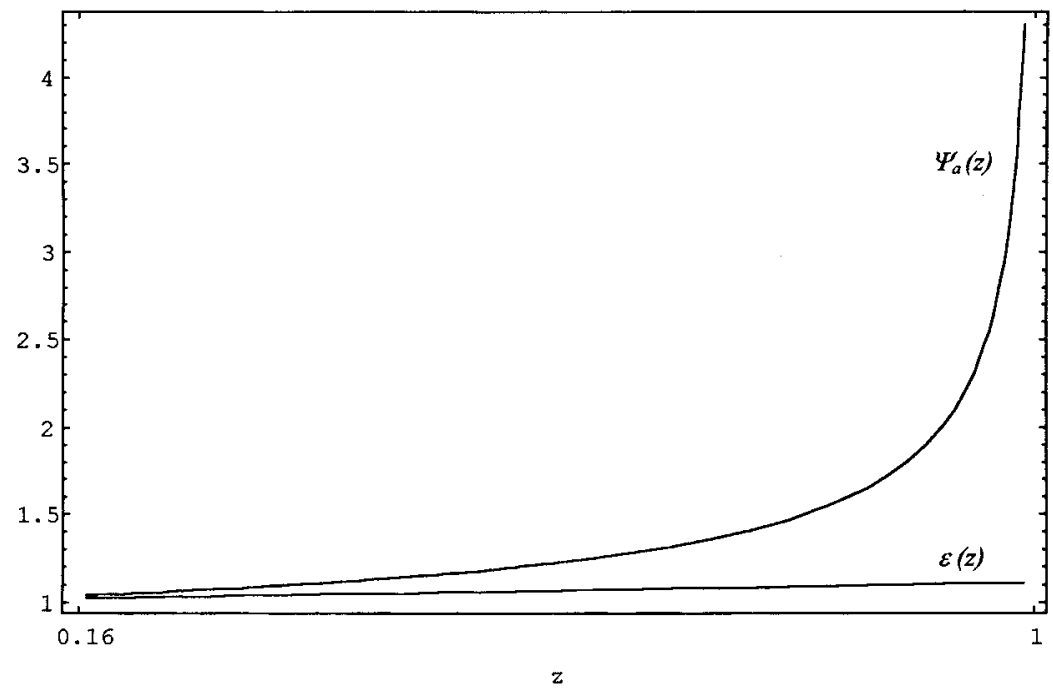

FIG. 1. The case of an attractive fixed point of the optimal policy at $1 / 1+\lambda$. The function $\varepsilon(z)$ is smaller than $\Psi_{a}(z)$ on the entire interval $[\lambda /(1+\lambda), 1)$. 
theta $(x)$

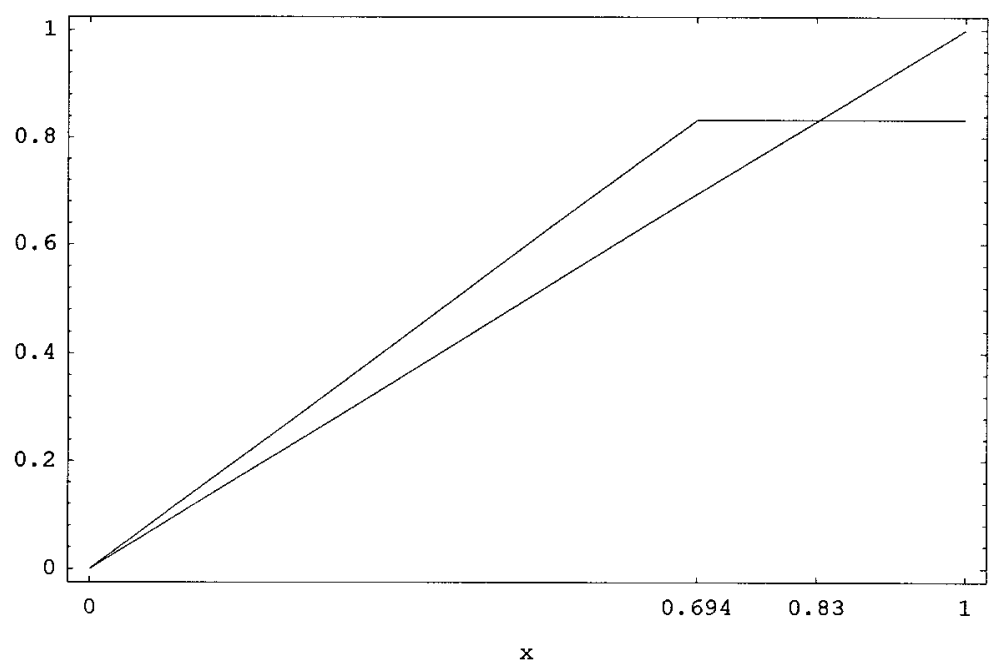

FIG. 2. The optimal policy for the case $\varepsilon(z)<\Psi_{a}(z) \forall z \in[\lambda /(1+\lambda), 1)$.

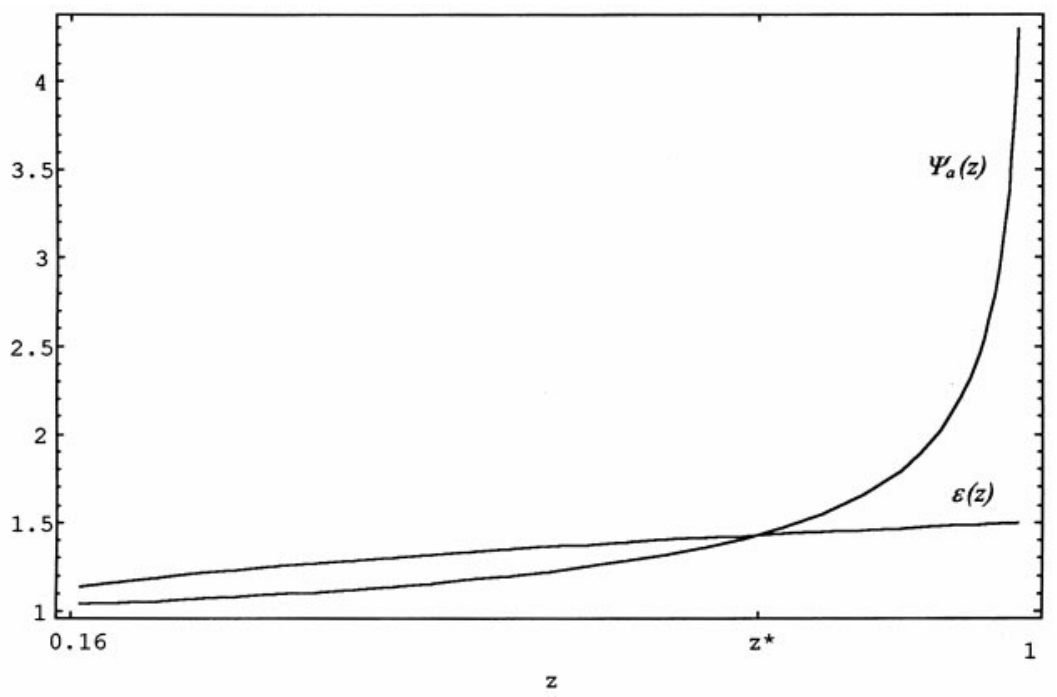

FIG. 3. The case of a stationary cycle of the optimal program. The period of the cycle is determined by the unique intersection point $z^{*}$ of $\varepsilon(z)$ and $\Psi_{a}(z)$, as shown in the figure. 
theta $(x)$

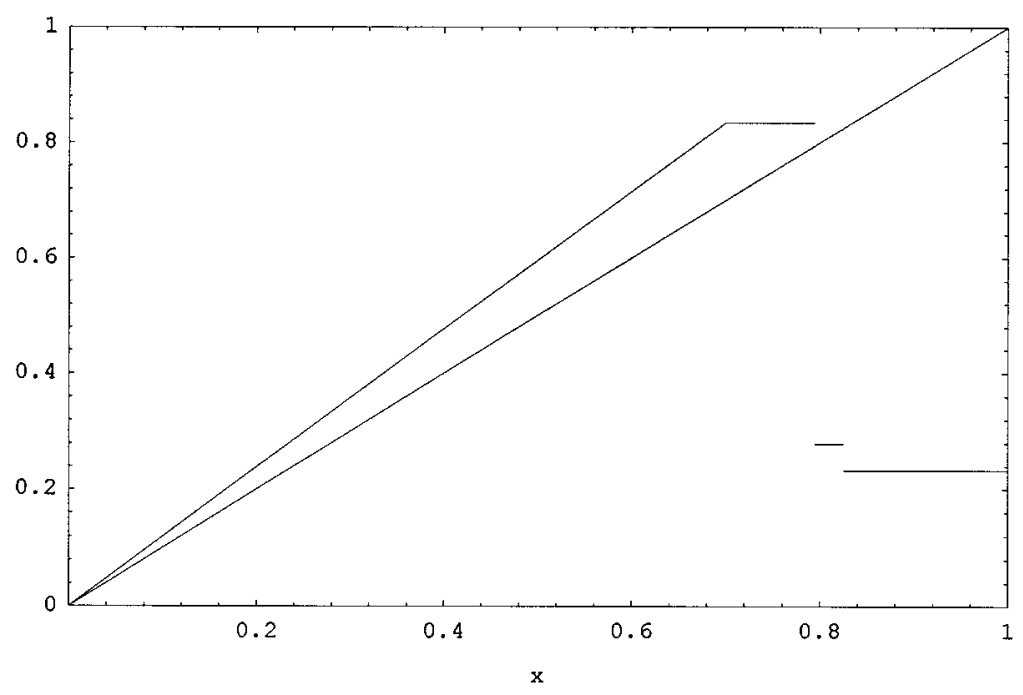

FIG. 4. The optimal policy for the case shown in Figure 3.

\section{EXTINCTION OF RESOURCES: THE SUBMARGINAL CASE}

Throughout this section we assume that $\delta(1+\lambda) \leqslant 1$, i.e. the growth factor of the resource is less than or equal to the reciprocal of the discount factor even when the whole potential of growth of the resource can be used. In these circumstances it seems to be reasonable that an extinction policy might be optimal. In Lemma 8 we show that immediate extinction is indeed optimal if the stock of resource is so high that not the full potential of growth can be exploited.

Lemma 8. Let $\delta(1+\lambda) \leqslant 1$, then $\theta(x)=0 \forall x \in[1 /(1+\lambda), 1]$.

Proof. Taking into account Lemma 1 we have to show $\theta(1 /(1+\lambda))=0$. Using the same arguments as we used in proving Proposition 2 and defining $g(z)$ again by (6) we conclude that showing $\operatorname{argmax}_{z \in[\lambda /(1+\lambda), 1]} g(z)=1$ establishes the claim of the Lemma. As $R(u)$ is assumed to be convex we have $\varepsilon(z)>1 \forall z \in[\lambda /(1+\lambda), 1]$ and, according to Lemma $7, \Psi_{a}(z) \leqslant 1$ $\forall z \in[\lambda /(1+\lambda), 1]$ (recall that $a \geqslant 1 \Leftrightarrow \delta(1+\lambda) \leqslant 1$ ). Thus we conclude that $g^{\prime}(z)>0 \forall z \in[\lambda /(1+\lambda), 1]$ which completes the proof.

Note that Lemma 8 implies that in the submarginal case an optimal sequence of controls contains at most two positive harvests. Either the first 
harvest leads to a complete extinction of the resource stock or the stock grows without any further harvest until it reaches the level $x=1 /(1+\lambda)$ (this follows from Lemma 3 ) and is then completely harvested. We will show that it depends on the curvature of $R(u)$ and on the initial stock of resource whether one or two harvests are optimal.

Contrary to the supramarginal case, the shape of the optimal policy on the interval $\left[0,1 /(1+\lambda)^{2}\right]$ depends on the properties of the immediate benefit function $R(u)$. Nevertheless, the next lemma shows that the number of possible elements of $\theta(x)$ can be restricted to three for $x \in\left[0,1 /(1+\lambda)^{2}\right)$ and to two for $x \in\left[1 /(1+\lambda)^{2}, 1 /(1+\lambda)\right)$.

Lemma 9. Let $\delta(1+\lambda) \leqslant 1$, then for $x \in\left(1 /(1+\lambda)^{k+1}, 1 /(1+\lambda)^{k}\right] k \geqslant 2$, one of the following three cases can occur: $(\alpha) \theta(x)=(1+\lambda) x,(\beta) \theta(x)=$ $1 /(1+\lambda)^{k},(\gamma) \theta(x)=0$. For $x \in\left[1 /(1+\lambda)^{2}, 1 /(1+\lambda)\right)$ only cases $(\beta)$ or $(\gamma)$ are possible.

Proof. We first consider the case where $x \in\left(1 /(1+\lambda)^{k+1}, 1 /(1+\lambda)^{k}\right]$ for some $k \geqslant 2$. According to Lemma 3 we have $\theta(x)=1 /(1+\lambda)^{n}$ for some integer $n \geqslant k$ or $\theta(x)=0$ or $\theta(x)=(1+\lambda) x$. Using Lemmata 3 and 8 we see that the payoff in the first case is $R\left((1+\lambda) x-\left(1 /(1+\lambda)^{n}\right)\right)+\delta^{n} R(1)$. Setting $y=1 /(1+\lambda)^{n}$ this reads $R((1+\lambda) x-y)+y^{a} R(1)$, where $y \in\left[0,1 /(1+\lambda)^{k}\right]$ and $a$ is given by (4). As $a \geqslant 1$ this expression is convex in $y$ which implies that the maximum of this function is either attained at $y=0$ or at $y=1 /(1+\lambda)^{k}$. Thus one of the cases $(\alpha)-(\gamma)$ holds true. For $x \in\left(1 /(1+\lambda)^{2}, 1 /(1+\lambda)\right)$ the same arguments apply but due to Corollary 1 we know that $\theta(x) \neq(1+\lambda) x$ which excludes case $(\alpha)$.

In Proposition 3 we give a complete characterization of optimal programs in the case where the average elasticity of $R(u)$ on every interval of the form $[u,(1+\lambda) u]$ is large enough to outweigh the "net loss" or the "opportunity costs" of leaving the stock unharvested, at least in the periods of "full growth".

Proposition 3. Let $\delta(1+\lambda) \leqslant 1$ and a again be given by (4). Assume that

$$
\frac{1}{\lambda u} \int_{u}^{(1+\lambda) u} \varepsilon(z) d z>a
$$

is satisfied for all $u \in(0,1 /(1+\lambda)]$, then there is a point $x^{*} \in\left(1 /(1+\lambda)^{2}\right.$, $1 /(1+\lambda))$ defined uniquely by

$$
R\left((1+\lambda) x^{*}\right)=R\left((1+\lambda) x^{*}-\frac{1}{1+\lambda}\right)+\delta R(1)
$$


such that for every $x \in\left(1 /(1+\lambda)^{k+1}, 1 /(1+\lambda)^{k}\right], k \geqslant 1$ the optimal program has one of the three following forms.

(i)] If $x<x^{*} /(1+\lambda)^{k-1}$ the optimal program is

$$
\left\{x,(1+\lambda) x, \ldots,(1+\lambda)^{k-1} x, \frac{1}{1+\lambda}, 0,0, \ldots\right\}
$$

(ii) If $x>x^{*} /(1+\lambda)^{k-1}$ the optimal program is

$$
\left\{x,(1+\lambda) x, \ldots,(1+\lambda)^{k-1} x, 0,0, \ldots\right\},
$$

(iii) If $x=x^{*} /(1+\lambda)^{k-1}$ both programs given above are optimal.

If $x>1 /(1+\lambda)$ the optimal program is given by

(iv) $\{x, 0,0, \ldots\}$.

In particular, all optimal programs have one of these forms if $\varepsilon(x)>a$ $\forall x \in[0,1]$.

Proof. According to Lemma 5 we have $\theta(x)=(1+\lambda) x \quad \forall x \in$ $\left(0,1 /(1+\lambda)^{2}\right]$. Thus we have $\theta\left(1 /(1+\lambda)^{2}\right)=1 /(1+\lambda)$ and by Lemma 8 it follows that $\theta(1 /(1+\lambda))=0$. Using that $\theta(x)$ is nonincreasing on $\left[1 /(1+\lambda)^{2}, 1 /(1+\lambda)\right]($ Lemma 2$)$ and Lemma 9 we conclude that there must exist a unique point $x^{*} \in\left(1 /(1+\lambda)^{2}, 1 /(1+\lambda)\right)$ with $\theta(x)=1 /(1+\lambda)$ $\forall x \in\left[1 /(1+\lambda)^{2}, x^{*}\right)$ and $\theta(x)=0 \forall x \in\left(x^{*}, 1 /(1+\lambda)\right]$. Due to continuity arguments the optimal policy at $x^{*}$ has to be set-valued, $\theta\left(x^{*}\right)=$ $\{0,1 /(1+\lambda)\}$, which implies that $R\left((1+\lambda) x^{*}\right)=R\left((1+\lambda) x^{*}-1 /(1+\lambda)\right)+$ $\delta R(1)$ must hold. The strict convexity of $R(x)$ guarantees that no other point than $x^{*}$ satisfies this equation. The calculation of the optimal programs is now straightforward. Starting with a stock $x$ no harvest takes place until the stock enters the interval $\left[1 /(1+\lambda)^{2}, 1 /(1+\lambda)\right]$. If $x<x^{*} /(1+\lambda)^{k-1}$ we have according to the argument given above $\theta\left((1+\lambda)^{k-1} x\right)=1 /(1+\lambda)$ and in the next period $\theta(1 /(1+\lambda))=0$. This yields exactly the optimal program given in (i). If $x>x^{*} /(1+\lambda)^{k-1}$ we have $\theta\left((1+\lambda)^{k-1} x\right)=0$ which yields (ii). In the case $x=x^{*} /(1+\lambda)^{k-1}$ the optimal policy at $(1+\lambda)^{k-1} x$ is set-valued yielding both programs presented above as optimal programs. The optimal program from $x>1 /(1+\lambda)$ follows directly from Lemma 8 . It is easy to see that the condition of the proposition is met whenever $\varepsilon(u)>a \forall u \in[0,1]$ holds.

The intuition behind this proposition is clear. Recall that the parameter $a$ denotes the value of the elasticity of the net benefit function for which the central planner would be indifferent between harvesting $u$ now and 
harvesting $(1+\lambda) u$ in the next period. If the average elasticity of $R(u)$ is larger than $a$, waiting for one period will pay off as long as the stock is below $1 /(1+\lambda)^{2}$. If the stock is above this level, waiting for one period would mean that in the next period the effective growth factor would be smaller than $1+\lambda$. Thus for stocks between $1 /(1+\lambda)^{2}$ and $1 /(1+\lambda)$ a trade-off between these two effects exists. For $x \leqslant x^{*}$ the "elasticity effect" is larger and the basic decision is to postpone extinction for one period. However, in order to exploit the whole growth potential of the resource, the stock is reduced to $x=1 /(1+\lambda)$. If $x>x^{*}$ the second effect is larger and the resource is driven extinct immediately. Note further that Proposition 3 implies that the fact whether one or two positive harvests are carried out in the optimal program depends on the initial stock of the resource. The whole interval $[0,1]$ is partitioned in infinitely many intervals which get infinitely small near 0 such that every second interval implies that one harvest is optimal if the initial stock lies within this interval and every other interval implies the optimality of two harvests.

In the next proposition we show that immediate extinction is optimal from every initial stock if the average elasticity of $R(u)$ is small enough.

Proposition 4. Let again $\delta(1+\lambda) \leqslant 1$, and a given by (4). Assume that for every point $u \in\left(1 /(1+\lambda)^{k+1}, 1 /(1+\lambda)^{k}\right], k \geqslant 1$

$$
\eta(u, i):=\frac{1}{\left((1+\lambda)^{i}-1\right) u} \int_{u}^{(1+\lambda)^{i} u} \varepsilon(z) d z \leqslant a
$$

holds for every $1 \leqslant i \leqslant k$. Then the immediate extinction policy $\theta(x)=0$ corresponding to the program

$$
\{x, 0,0, \ldots\}
$$

is optimal for every $x \in[0,1]$. In particular, this holds true if $\varepsilon(x)<a$ $\forall x \in[0,1]$. If inequality (12) is strict for all $1 \leqslant i \leqslant k$ then this program is the unique optimal program from every $x$.

Proof. Putting together the claims of Lemmata 3 and 8 we realize that in any optimal sequence of controls $\left\{\hat{u}_{t}\right\}_{t=0}^{\infty}$ at most two positive controls may appear. After these two controls have been applied the absorbing state zero will be reached. Let us assume that the optimal sequence of controls has two positive elements, $\hat{u}_{n_{1}}$ and $\hat{u}_{n_{2}}$ with $0 \leqslant n_{1}<n_{2}$. Obviously, $\hat{u}_{n_{2}}=$ $(1+\lambda)^{n_{2}-n_{1}}\left((1+\lambda)^{n_{1}+1} x-\hat{u}_{n_{1}}\right)$. Thus the corresponding program yields a payoff of 


$$
\begin{array}{rl}
\delta^{n_{1}} & R\left(\hat{u}_{n_{1}}\right)+\delta^{n_{2}} R\left(\hat{u}_{n_{2}}\right) \\
= & \delta^{n_{1}} R\left(\hat{u}_{n_{1}}\right)+\delta^{n_{2}} R\left((1+\lambda)^{n_{2}-n_{1}}\left((1+\lambda)^{n_{1}+1} x-\hat{u}_{n_{1}}\right)\right) \\
= & \delta^{n_{1}} R\left(\hat{u}_{n_{1}}\right)+\delta^{n_{2}}(1+\lambda)^{\left(n_{2}-n_{1}\right) \eta\left((1+\lambda)^{n_{1}+1} x-\hat{u}_{n_{1}}, n_{2}-n_{1}\right)} R\left((1+\lambda)^{n_{1}+1} x-\hat{u}_{n_{1}}\right) \\
& \leqslant \delta^{n_{1}} R\left(\hat{u}_{n_{1}}\right)+\delta^{n_{1}}\left(\delta^{n_{2}-n_{1}}(1+\lambda)^{a\left(n_{2}-n_{1}\right)}\right) R\left((1+\lambda)^{n_{1}+1} x-\hat{u}_{n_{1}}\right) \\
& =\delta^{n_{1}} R\left(\hat{u}_{n_{1}}\right)+\delta^{n_{1}} R\left((1+\lambda)^{n_{1}+1} x-\hat{u}_{n_{1}}\right) \\
& <\delta^{n_{1}} R\left((1+\lambda)^{n_{1}+1} x\right),
\end{array}
$$

where we used Lemma 4, the assumption made in (12) and the fact that $R(u)$ is convex. The last line gives exactly the payoff yielded by the program $\left\{\tilde{u}_{t}\right\}_{t=0}^{\infty}$ with $\tilde{u}_{t}=0 \forall t \neq n_{1}, \tilde{u}_{n_{1}}=(1+\lambda)^{n_{1}+1} x$. Thus for every program with two optimal controls there exists a program with only one optimal control which gives a higher discounted utility. Focusing on programs with only one positive control we realize that any such program yields a discounted utility of $\delta^{n} R\left((1+\lambda)^{n+1} x\right)$ where $n$ is the period with the positive control. Using again Lemma 4 and the assumptions made in the proposition we get

$$
\begin{aligned}
\delta^{n} R\left((1+\lambda)^{n+1} x\right) & =\delta^{n}(1+\lambda)^{n \eta((1+\lambda) x, n)} R((1+\lambda) x) \\
& \leqslant \delta^{n}(1+\lambda)^{a n} R((1+\lambda) x) \\
& =R((1+\lambda) x)
\end{aligned}
$$

Thus immediate harvesting of the whole stock gives the maximal attainable payoff. For the optimal policy this implies $0 \in \theta(x) \forall x \in\left[0,1 /(1+\lambda)^{2}\right)$. The optimal policy is single-valued, namely $\theta(x)=0$ on $\left(1 /(1+\lambda)^{2}\right.$, $1 /(1+\lambda)]$ as there is no selection of $\theta(x)$ which is increasing on this interval (Lemma 2). Together with Lemma 8 this yields $0 \in \theta(x) \forall x \in$ $[0,1]$. Thus immediate extinction is optimal for any stock $x \in[0,1]$. If the inequality (12) is strict for all $i \geqslant 1$ the reasoning above shows that immediate harvesting yields a strictly higher payoff than any other program and we have $\theta(x)=0 \forall x \in\left[0,1 /(1+\lambda)^{2}\right]$. Thus the optimal policy is singlevalued for every $x \in[0,1]$ which means that any optimal program is unique.

Propositions 3 and 4 give no complete characterization of optimal programs in the submarginal case. The question is left open how the optimal programs look like if the elasticity crosses the line $\varepsilon(z)=a$ within $(0,1)$. We have carried out an analysis of the cases where only one crossing occurs but the derived criteria and also the optimal policies turned out to be rather intricate and we abstain from presenting them here. 


\section{DISCUSSION}

In this paper we have analyzed a time-discrete one-dimensional model of optimal resource exploitation where the reproduction function is concave but the net benefit function exhibits a nonconcavity. In agreement with the cases of a concave or linear objective function we come to the conclusion that the fact whether an extinction or a conservation policy is optimal is determined by the value of the product of the discount factor $\delta$ and the growth factor $(1+\lambda)$. We have shown that in the supramarginal case, i.e., $\delta(1+\lambda)>1$, the long term behavior of an optimal program is independent from the initial stock but depends on the elasticity of the immediate benefit function. To our knowledge no such characterization of optimal programs in aggregative models where the return function has non-constant elasticity has been given so far. Furthermore, it was illustrated that cycles of periods larger than one may describe the long run behavior of an optimal program, and that the cycle length can be computed by using rather simple means. It is worth mentioning that several authors noted earlier that it is exactly such a complete characterization of optimal cyclical policies on which a better understanding of problems with nonconcavities must rest (see e.g. $[16,21])$.

Some of the results in the existing literature still hold in our case. Clark [6] has shown that in the case of a linear return function and a supramarginal resource any optimal program converges to a fixed point. This result remains valid in our setup if the elasticity of the (convex) benefit function is smaller than some constant depending on the discount and growth rate. In particular it holds for "almost" linear benefit functions, and we would like to point out the connection to results on most rapid approach paths in accumulation problems, see Spence and Starett [36]. In the submarginal case, i.e. $\delta(1+\lambda) \leqslant 1$, all results from the existing literature are confirmed, namely that every optimal program leads to eventual extinction $([6,11,23])$. The only difference is that in our model the elasticity of $R(u)$ determines whether the extinction is immediate or not.

The results presented in this paper may be linked to results from a quite different field, namely the theory of inventory control. It has been shown that in finite horizon inventory models with concave ordering costs (composed of a unit cost plus a reorder cost) a so-called $[s, S]$ policy is optimal (see $[17,33])$. This means that whenever the stock decreases below the amount $s$ it is build up to the level $S$. Optimal programs in our model may be seen as some kind of inverted $[s, S]$ policy (see also $[16,31,37]$ ). In the supramarginal case, whenever the stock of resource has reached the level $x=1 /(1+\lambda)$ it is exploited to some level $x=1 /(1+\lambda)^{m}$. Also the extinction policies in the submarginal case can be written in this way: For immediate extinction we have an inverted $(0,0)$ policy, for eventual 
extinction a $(0,1 /(1+\lambda))$ policy. Hence optimal policies can only be characterized by a pair of critical numbers, whereas for models with concave or linear immediate return function a single critical number is sufficient to characterize the optimal control policy (see also [1]).

We conclude our discussion with pointing out some further topics of research. The results presented in this paper have been derived for the deterministic framework. In recent years bioeconomics has been concerned with the behavior under uncertainty. The effects of uncertainty on the results of bioeconomic analysis investigated so far are rarely unambiguous though, and raised the question of whether uncertainty is significant (for a survey, see [1]). In several cases deterministic policies has been proven to be reasonably good substitutes for stochastic ones. Since one cannot easily generalize from existing results this remains to be investigated for the class of models presented here. Furthermore, following the rather complete characterization for a continuous-time framework (see [13]) an interesting extension of our model would be to use a continuously differentiable (concave or S-shaped) growth function instead of our piecewise linear one, or to analyze a model under the assumption of an S-shaped immediate return function (see also $[18,19]$ for some numerical results). It is also indicated to assume that either there is some kind of critical stock such that there is no growth as long as the stock of resource is below this critical level (see $[9,26,27]$ ), or that there is some potential of biological regeneration even if the stock is driven to extinction. In any case, from a mathematical point of view it is desirable to have a general characterization of the behavior of optimal programs for different constellations of the growth function and the immediate return function, especially for the poorly understood cases of a nonconcavity in the objective function (see $[12,22]$ for a further discussion). In this paper we have analyzed one such constellation.

\section{REFERENCES}

1. P. Andersen and J. G. Sutinen, Stochastic bioeconomics: A review of basic methods and results, Mar. Res. Econ. 1 (1984), 117-136.

2. J. Benhabib and K. Nishimura, Competitive equilibrium cycles, J. Econ. Theory 40 (1985), 284-306.

3. T. Bjørndal, Production economics and optimal stock size in a North Atlantic fishery, Scand. J. Econ. 89 (1987), 145-164.

4. T. Bjørndal and J. M. Conrad, The dynamics of an open access fishery, Can. J. Econ. 20 (1987), 74-85.

5. T. Bjørndal, J. M. Conrad, and K. G. Salvanes, Stock size, harvesting costs, and the potential for extinction: The case of sealing, Land Econ. 69 (1993), 156-167.

6. C. W. Clark, Economically optimal policies for the utilization of biologically renewable resources, Math. Biosci. 12 (1971), 245-260. 
7. C. W. Clark, "Mathematical Bioeconomics: The Optimal Management of Renewable Resources," Wiley, New York, 1990.

8. W. S. Comanor and T. A. Wilson, “Advertising and Market Power," Harvard Univ. Press, Cambridge, 1975.

9. J. M. Conrad and C. W. Clark, "Natural Resource Economics," Cambridge Univ. Press, Cambridge, 1987.

10. K. Cowling, J. Cable, M. Kelly, and T. McGuiness, "Advertising and Economic Behavior," Holmes \& Meier Publishers, New York, 1975.

11. W. D. Dechert and K. Nishimura, A complete characterization of optimal growth paths in an aggregated model with a non-concave production function, J. Econ. Theory 31 (1983), 332-354.

12. H. Dawid, M. Kopel, and G. Feichtinger, Complex solutions of nonconcave dynamic optimization models, Econ. Theory 9 (1997), 427-439.

13. G. Feichtinger and R. F. Hartl, "Optimale Kontrolle ökonomischer Prozesse," Walter de Gruyter, Berlin, 1986.

14. R. Hannesson, Fishery dynamics: A North Atlantic cod fishery, Can. J. Econ. 8 (1975), 151-173.

15. R. Hannesson, Bioeconomic production function in fisheries: Theoretical and empirical analysis, Can. J. Fish. Aquat. Sci. 40 (1983), 968-982.

16. D. L. Jaquette, A discrete-time population-control model with setup cost, Oper. Res. 22 (1974), 298-303.

17. S. Karlin, Optimal inventory policy for the Arrow-Harris-Marschak Dynamic model, in "Studies in the Mathematical Theory of Inventory and Production" (K. J. Arrow, S. Karlin, and H. Scarf, Eds.), pp. 135-154, Stanford Univ. Press, Stanford, 1958.

18. T. R. Lewis, Exploitation of a renewable resource under uncertainty, Can. J. Econ. 14 (1981), 422-439.

19. T. R. Lewis, "Stochastic Modeling of Ocean Fisheries Management," University of Washington Press, Seattle/London, 1982.

20. T. R. Lewis and R. Schmalensee, Nonconvexity and optimal exhaustion of renewable resources, Internat. Econ. Rev. 18 (1977), 535-552.

21. T. R. Lewis and R. Schmalensee, Non-convexity and optimal harvesting strategies for renewable resources, Can. J. Econ. 12 (1979), 677-691.

22. D. Levhari, R. Michener, and L. J. Mirman, Nonconcave dynamic programming: An example, in "Essays in the economics of renewable resources" (L. J. Mirman and D. F. Spulber, Eds.), pp. 79-93, North-Holland, Amsterdam, 1982.

23. M. Majumdar and T. Mitra, On optimal exploitation of a renewable resource in a nonconvex environment and the minimum safe standard of conservation, working paper 223, Cornell University, New York, 1980.

24. M. Majumdar and T. Mitra, Intertemporal allocation with a non-convex technology: The aggregative framework, J. Econ. Theory 27 (1982), 101-136.

25. M. Majumdar and T. Mitra, Dynamic optimization with a non-convex technology: The case of a linear objective function, Rev. Econ. Studies 50 (1983), 143-151.

26. T. Mitra and H. Y. Wan Jr., Some theoretical results on the economics of forestry, Rev. Econ. Studies 52 (1985), 263-282.

27. P. A. Neher, "Natural Resource Economics-Conservation and Exploitation," Cambridge Univ. Press, Cambridge, 1990.

28. K. Nishimura and M. Yano, Optimal chaos, nonlinearity and feasibility conditions, Econ. Theory 4 (1994), 689-704.

29. K. Nishimura and M. Yano, Nonlinear dynamics and chaos in optimal growth: An example, Econometrica 4 (1995), 981-1001. 
30. L. J. Olson and S. Roy, On conversation of renewable resources with stock-dependent return and non-concave production, J. Econ. Theory 70 (1996), 133-157.

31. W. J. Reed, A stochastic model for the economic management of a renewable animal resource, Math. Biosci. 22 (1974), 313-337.

32. W. E. Ricker, Stock and recruitment, J. Fish. Res. Board Can. 11 (1954), 559-623.

33. H. Scarf, The optimality of $(S, s)$ policies in the dynamic inventory problem, in "Mathematical Methods in the Social Sciences" (K. J. Arrow, S. Karlin, and P. Suppes, Eds.), pp. 196-202, Stanford Univ. Press, Stanford, 1960.

34. R. Schmalensee, "The Economics of Advertising," North-Holland, Amsterdam, 1972.

35. A. K. Skiba, Optimal growth with a convex-concave production function, Econometrica 46 (1978), 527-539.

36. M. Spence and D. Starett, Most rapid approach paths in accumulation problems, Internat. Econ. Rev. 16 (1975), 388-403.

37. D. F. Spulber, Adaptive harvesting of a renewable resourc and stable equilibrium, in "Essays in the Economics of Renewable Resources" (L. J. Mirman and D. F. Spulber, Eds.), pp. 117-139, North-Holland, Amsterdam, 1982.

38. N. L. Stokey and R. E. Lucas (with E. C. Prescott), "Recursive Methods in Economic Dynamics," Harvard Univ. Press, Cambridge, 1989.

39. O. Tavhonen and S. Salo, Nonconvexities in optimal pollution accumulation, J. Environ. Econ. Management 31 (1996), 160-177. 